\title{
Analysis of Packet Loss in Next Generation Networks Using Large Derivation
}

\author{
Liji P I *and S Bose**
}

\begin{abstract}
The Next Generation Wireless Networks is a packet based IP network that supports anytime, anywhere service and provides Always Best Connected(ABC) state. Most of the aggregated traffic in IP based network is real time multimedia and this would not guarantee best effort of service, so the performance gets degraded in the networks. Main feature of ubiquitous NGN wireless communication system is seamless mobility. Mobile terminal in the network will be roaming in the vicinity of the heterogeneous wireless network. According to the Received Signal Strength (RSS) the mobile terminal in the network will handover from one technology to another. As there is frequent handoff from one technology to another can results in performance degradation .If enough resource is not available packet will be blocked or dropped in the network this can degrade the performance in the network. It is desired to have a resource allocation scheme which can satisfy the QoS constraints while maximizing the utilization of the network resources with minimizing the packet loss and delay in the network. The mobile terminal in the network carry integrated real time multimedia data (eg: Video streaming, videoconferencing, IPTV and on-line gaming) and this type of service should guarantee high Quality of Service(QoS) with minimum delay ,packet loss, and jitters. Traffic model can be used as input to analysis resource allocation strategies. Traffic in the network can be modeled in such a way that it should allocate resource efficiently and then reduce queuing delay, packet loss and jitters in the NGN environment to meet the QoS given by the Service Level Agreement(SLA). The high variability data in the network is bursty in nature and conventional traffic modeling like Poisson or Markovian process is inappropriate to model traffic in the networks. The burstiness can be represented as Self similarity with long range dependency. FARIMA a self similar time series model can represent the higher priority traffic in the network with short range and long range dependency and using large derivation packet loss in the network is analyzed.
\end{abstract}

Keywords — QoS, Packet loss, Self similarity, Large Derivation.

\section{I.INTRODUCTION}

Next generation networks are of ubiquitous wireless communications systems and provided with seamless highquality wireless services for the device. The connectivity can be achieved through the IP protocols[2]. The mobility in the NGN is to provide a seamless service to mobile users so that the user can move between heterogeneous networks regardless of location. If resource are not sufficient in the network call will be dropped or delayed thus the Quality of Service defined by the SLA (Service Level Agreement) will be affected. If the

\footnotetext{
*Research Scholar, Department of Computer Science \& Engineering, Anna University, Chennai

**Associate Professor Department of Computer Science \& Engineering, Anna University, Chennai
}

resources are allocated dynamically using traffic prediction, performance in the network can be improved. The aggregated traffic in the network shows high variability auto-correlation decays to zero at a slower rate than exponential. This high variability traffic can be represented with self similarity fractal property. So conventional traffic models like Poisson or Markovian model are inappropriate to represent bursty traffic and this burstiness can be represented as a self similarity model with long range dependency. Self similarity with fractal behavior can be represented as Fractional Gaussian Noise (example of an exactly self similar) [7][8]or Fractional ARIMA (an asymptotically self-similar process). Highly correlated and higher priority VBR traffic in network in the network can be represented with FARIMA time series model with parameters (p,d-1/ $\alpha, q)$.FARIMA can represent the actual data and this can predict the future traffic from present information and allocate resource on demand either as peak value or mean value. So congestion in the network can be reduced .Packet loss one of the major factor that affect the QoS performance in the network can be analyzed with large derivation theory.

The rest of the paper is organized as follows. Section 2 briefly describes the characteristics of self similarity along with long range dependency and heavy tailed distribution in Section 3,introduction to FARIMA and traffic prediction using time series model; in section 4,bandwidth allocation for the network ; in Section 5 packet loss analysis, Section 6 concludes this paper.

\section{II.BACKGROUND OF SELF- SIMILARITY, LONG RANGE DEPENDENCY AND HEAVY TAILED DISTRIBUTIONS}

\subsection{Self-similarity Traffic Model}

Second-order statistics are statistical properties that capture burstiness or variability in the real time multimedia traffic. Leland et al [10] propose that Ethernet traffic can be modelled by self-similar possesses and possesses long-range dependence(LRD).Self-similarity a fractal property can describes burstiness[5][8],aggregated traffic in the network can be represented as a stochastic self similar process if $\mathrm{X}(\mathrm{at})=\mathrm{a}^{\mathrm{H}} \mathrm{X}(\mathrm{t})$, where $\mathrm{H}$ is the Hurst parameter that represent the self similarity and as $\mathrm{H}->1$,burstiness in the traffic will increases. The Hurst exponent estimate is conducted with the help of three widely spread approaches, the correlation function slope analysis in log-log scale, the variance-time plot analysis and $\mathrm{R} / \mathrm{S}[]$. The wide sense stationary stochastic process $\mathrm{X}(\mathrm{t})$ has mean $\mu_{x}$,variance $\sigma^{2}$ and autocorrelation 
function $\rho_{x(k)}$.The autocorrelation function can be represented as

$$
\rho_{x(k)} \approx k^{-\beta} L_{1}(k), k \rightarrow \infty
$$

where $0<\beta<1$ and $L_{1}$ slowly vary to infinite

If $\rho^{m}{ }_{x}(k)=\rho_{x}, m \rightarrow \infty$, the process is called asymptotically second-order self-similar and for exactly second order self similar processes $\rho^{m}{ }_{x}(k)=\rho_{x}, k \geq 0$, this implying that the sum of auto correlation diverge. The covariance function decays hyperbolically Main features of self similarity are 1 . Slowly decaying variance 2 . the auto correlation is not summable 3.spectral density obey power law in the origin [2].Self-similarity along with long range dependency and the heavy tailed distribution has got significant impact in the queuing analysis of the network.

\subsection{Long Range Dependency and Heavy Tailed Distribution}

The high speed traffic in the network exhibit a property of correlation over a wide range of time scale and this can be represented as long memory or long range dependency process. Statistical analysis of bursty real time data collected from networks shows the property of self- similarity with long range dependency. Let $X_{t}=1,2, \ldots$ be a stochastic self similarity process with long range dependency and the autocorrelation function can be represented as $\rho(k)=\frac{\operatorname{Cov}\left(x_{t} x_{t}+k\right)}{\operatorname{var}\left(x_{t}\right)}$.If this function is not summable (e.g., when it decays hyperbolic decay), then it is referred as a long range dependency process with $\sum_{j=0}^{\infty} \rho(k)=\infty$ If autocorrelation function is summable then process is a short range dependent as shown in fig : 1 The main characterize of LRD are:1. The autocorrelation function not absolutely summable 2 . The spectral density function becoming unbounded as the frequency tends to zero. The aggregated traffic from the multiple sources exhibits a Long Range Dependence and this is a statistical phenomenon observed in time series.

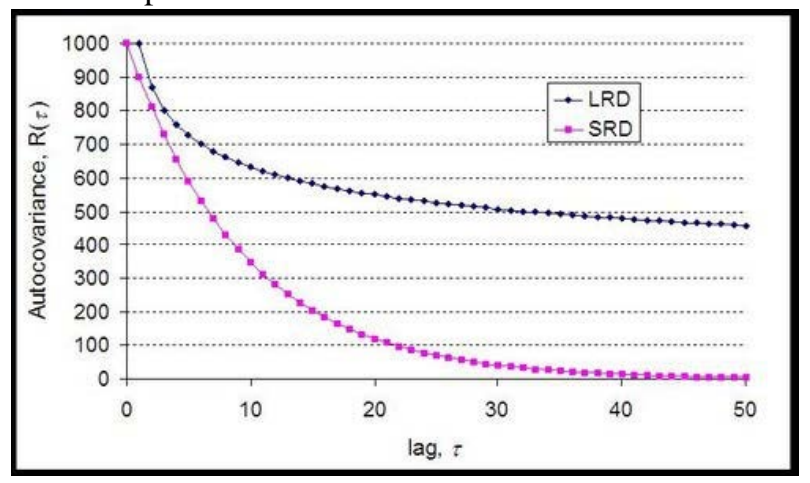

Fig. 1 Autocorrelation for Short range and Long range Dependency

Major cause of LRD in network traffic is due to infinite variances and high variability. The high variability non Gaussian bursty traffic can be represented as heavy tailed distributions .The high variability shows non Gaussian bursty traffic possess heavy tailed marginal distributions. The tail of the distribution decays much more slowly than in the case of an exponential distribution. The Pareto,Weibull and lognormal are some examples of a random variable drawn from a heavytail distribution which possess infinite variance or infinite mean depending on the value of the tail parameter $\alpha$. Heavy tailed distribution shows self similarity along with long range dependency [3], [4] and the tail of the distribution decays hyperbolically as $\mathrm{P}[\mathrm{X}>\mathrm{x}] \approx x^{-a}$ where $\mathrm{X}$ has a distribution with a heavy tail with tail index $\alpha$ and the distribution will be skewed to left as shown in fig:2

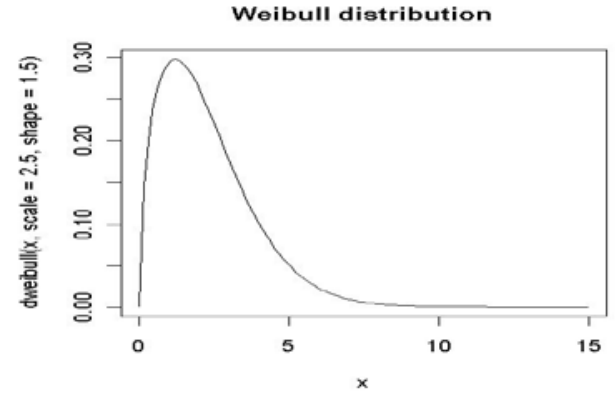

(a)

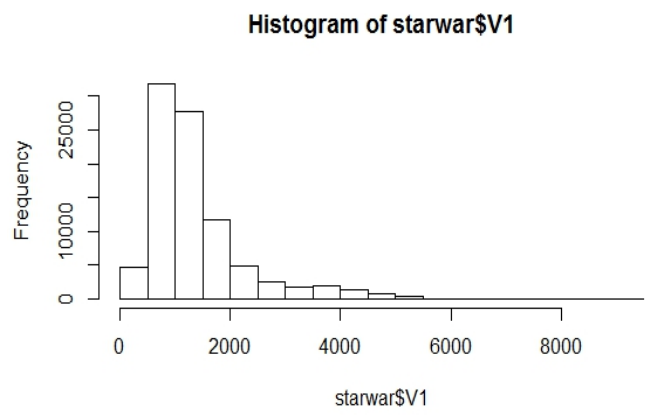

(b)

Fig:2 a. Heavy Tailed Distribution

b.Histogrm Representation for heavy tailed distribution

The high variability heavy tailed distributed self- similar VBR traffic in the next generation network can be modeled as Fractional Gaussian Noise, FARIMA or M/G/ $\infty$ model. The $\mathrm{M} / \mathrm{G} / \infty$ model can used to model the bursty traffic it is able to model both short range and long range dependence behaviors, inadequacies in modeling the marginal distribution [7],FGN will not able to model the short range lag in the VBR traffic. There for FARIMA is better choose for VBR traffic model [8].FARIMA model can represent both short range dependency and long range along with heavy tailed distribution traffic with parameters $(p, q)$ and $d-1 / \alpha$ [7] [8]. The heavy-tailed behavior of marginal distribution and the sub-exponential decay of autocorrelation function exhibited by video traffic have a significant impact on queuing performance [1], [8], hence real time traffic with high persistence had to be modeled

\section{Traffic Modeling And Prediction Using Time SERIES MODELS}

F-ARIMA is an extension of classic ARMA time series model and this is flexible to model both short term and long term correlation structure of a series[][8].Both lower frequency 
components as well as higher frequency components can be fitted using one or two parameters of FARIMA and long memory that can explicitly account for persistence to incorporate the long term correlation in the data. FARIMA $(\mathrm{p}, \mathrm{d}, \mathrm{q})$ process. FARIMA process is a standard given by ARIMA process where $\mathrm{d}$ degree of differencing .The FARIMA (0, d, 0) sequence showing the long-range dependence is generated $0.5<\mathrm{d}<1$.For stationary and invertibility it is assumed that all roots of $\theta(p) \phi(q)=0$ are outside the unit circle and $|\mathrm{d}|<0.5$. The series is strongly persistent or antipersistent according as $0<\mathrm{d}<0.5$ or $-0.5<\mathrm{d}$ $<0$. The special case where $\mathrm{p}=\mathrm{q}=0$ is known as fractionally differenced white noise(FDN). Real time multimedia traffic measurements exhibits both long range and short range dependency along with heavy tailed distribution can be represented with FARIMA(p,d-1/ $\alpha, q)$ time series $X=X(k), k \epsilon$ $\mathrm{Z}$, is define as

$$
\begin{gathered}
\phi(\mathrm{B}) \nabla^{d} \mathrm{X}_{\mathrm{t}}=\theta_{1}(\mathrm{~B}) \mathrm{a}_{\mathrm{n}} \\
\text { where } \theta(\mathrm{B})=\theta_{1}(\mathrm{~B})+\theta_{2}(\mathrm{~B})^{2}+\ldots+\theta p(\mathrm{~B})^{\mathrm{p}} \text { and } \\
\phi(\mathrm{B})=\phi_{1}(\mathrm{~B})+\phi_{2}(\mathrm{~B})^{2}+\ldots+\phi_{\mathrm{q}}(\mathrm{B})^{9} \text { where } \theta \text { and }
\end{gathered}
$$

$\phi$ are polynomials of order $p$ and $q$ respectively and $a_{n}$ is a white noise standard normal random variables. The lag operator is defined as $\nabla^{d} \mathrm{X}_{\mathrm{t}}=(1-\mathrm{B}) \mathrm{X}_{\mathrm{t}}=\mathrm{X}_{\mathrm{t}}-\mathrm{X}_{\mathrm{t}-1}$.The stochastic FARIMA traffic model techniques involve the fitting of traffic marginal distribution and correlation structure. The linear prediction can be done FARIMA traffic model and with minimum mean square error.

Steps for Prediction algorithm for a network traffic stream.

Step 1: From the QoS requirements of a particular network, determine the value of $u$ and $\xi$

Step 2:Estimate the parameters of the FARIMA model

Step 3.Calculate the residuals from the fitted model and obtain the 100uth percentile of the distribution of the residuals. Call it $\xi$

Step 4. Obtain the minimum mean square error forecast for $X_{t+h}$ based on the FARIMA model.

Step 5. The forecast for the 100th percentile is $\hat{\boldsymbol{X}}_{t+h}=X_{t+h}+\xi_{\mu}$

\section{DYNAMIC BANDWIDTH ALLOCATION}

The IP traffic in the next generation network is bursty in nature. The queuing analysis of long persistence bursty traffic causes large queues, large delays and the network performance will get degraded[9]. This will have a negative impact on the bursty real time VBR video traffic with stringent Quality of Service (QoS) parameters. The active mobile terminal roaming in the integrated heterogeneous network will keep search for high bandwidth. If enough resource is allocated in advance, call will be accepted. Certain admission policy should be ensured for a new connection or handoff connection in order to meet the QoS requirement for the traffic. To meet the QoS constraints in the network, high utilization of resource is needed. If allocation scheme allocates bandwidth equal to the peak rate is not desirable because a significant amount of bandwidth may be wasted due to the bursty nature of the VBR video traffic and mean value allocation may results in traffic congestion in the network. The Call admission control determine whether to accept or reject new call or handoff call and the bandwidth allocation should be neither average nor peak bandwidth allocation. It should have an effect value in between the average and peak value. Effective bandwidth can be considered for call admission control and the bandwidth allocated in the network. The resource in the network can be allocated on demand so as to achieve high utilization with less packet loss, delay and variance .The dynamic bandwidth allocation strategy can be used to allocate bandwidth on demand. In order to reserve network resource in advance, network should use current and past information to predict the future behavior of traffic. Prediction can allocate bandwidth dynamically. If we are able to predict $\mathrm{N}$ frame in advance and allocate the resource for the $\mathrm{N}$ frame, network performance can be improved. Parsimonious FARIMA can predict future N frame from present and past history of traffic

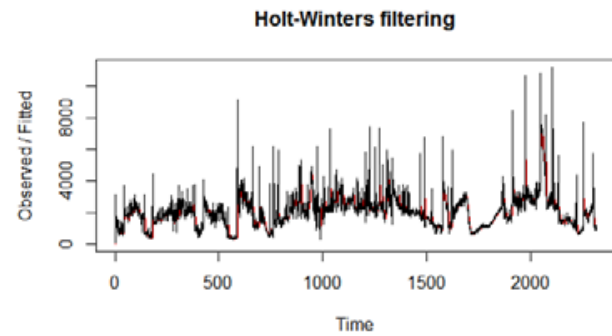

Forecasts from ARFIMA $(2,0.36,1)$

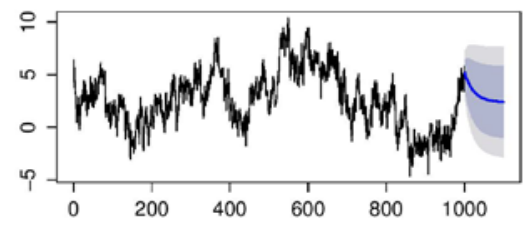

Fig. 3:a. Traffic model with best fit b. Forecast with FARIMA/ARFIMA

Traffic models are analyzed based on goodness-of-fit. To evaluate goodness-of-fit, one needs to define metrics that determine how "close" the model is to the actual data [8].Traffic model will best fit for real time with parameters $(2,0.36,1)$ and forecast the traffic as shown in fig. These metrics have to be directly related to the performance measures that are needed to be predicted from the model. The goodness-of-fit used in this article is based on the ability of the model to capture marginal distributions, auto-correlation structure, and ultimately predict delays and packet loss probabilities. 


\section{V.ANALYSIS OF PACKET LOSS WITH LARGE DERIVATION}

Packet loss in the network e measure of quality of service is loss probability, which can be analyzed using the theory of large deviations. When congestion occurs in the link, queues in routers build up to create long delays and packet losses that will decrease the quality of service (QoS) provided to the users[6][1]. In the case of 'conventional' traffic packet losses due to congestion are expected to have less impact on the QoS than in the case of bursty traffic where a burst of packets arriving at a full router is likely to be partially or entirely lost, this situations persist may harm the performance. The tail of the steady state queue length distribution $P\{Q>x\}$ is a fundamental measure of network congestion. For analysis of packet loss aggregated traffic is modeled by a fluid queue serving a large number of input processes. The packet loss probability in the queue of size $\boldsymbol{X}_{\text {can }}$ be mapped to $P\{Q>x\}$ and queuing analysis is based on Lindley`s equation queue converge to a steady state process.

$$
Q_{t}=\left(Q_{t-1}-A_{t}-S_{t}\right)^{+}
$$

Where $\mathrm{x}^{+}=\max (\mathrm{x}, 0)$

For single server with a FIFO queue the amount of work $A_{s, t}$ that arrived to be processed in the time interval $[\mathrm{s}, \mathrm{t})$ and $S_{s, t}$ the amount of work processed in the time interval $[\mathrm{s}, \mathrm{t}) \quad$ workload $\quad W$ is defined by $W_{t}=A_{-t-0}-S_{-t-0}$ and the queue length is given by $Q=\sup W_{t}$

$t \geq 0 \quad$ and the queue length can be characterized by large derivation property of the work load.

\section{CONCLUSION}

Real time VBR bursty network traffic is complex in next generation network, as it exhibits strong dependence. Selfsimilarity traffic is different from conventional traffic models such as Poisson and Markovian processes are not appropriate for real time traffic modeling. To maintaining high utilization of the bandwidth is the main objective for efficient traffic management, which includes CAC, policing, scheduling, buffer management, and congestion control etc. The high variable and highly correlated VBR traffic in the network can be modeled with self similarity models like FARIMA or FGN. Mostly VBR traffic in the network exhibits both short range and long range dependency along with heavy tailed distribution can be modeled with FARIMA time series model with parameter (p, d, q). FARIMA time series traffic model can predict traffic and allocate bandwidth dynamically. This model is flexible enough to parsimoniously capture the statistical property of traffic can allocate bandwidth on demand and mathematically analysis the queuing performance. Congestion in the network can be calculated with tail distribution using asymptotic behavior and packet loss in the network can be analysed with large derivation.

\section{REFERENCES}

[1] Xiaolong Jin,Geyong Min, Speros Velentzas, and Jianmin Jiang“Quality of-Service Analysis of Queuing Systems with LongRange-Dependent IEEE Transaction on Wireless Communication, Vol. 11, No. 2,pp 562-570,February 2012 http://dx.doi.org/10.1109/TWC.2011.120511.100867

[2] Yu Cheng ,W. Zhuang and L Wang "Calculation of Loss Probability in a Finite Size Partitioned Buffer for Quantitative Assured Service "IEEE Transaction on Communication ,Vol.55,No.9 pp.17571771,September 2007 http://dx.doi.org/10.1109/TCOMM.2007.904394

[3] Ian F. Akyildiz, Jiang Xie and Shantidev Mohanty,” A Ubiquitous Mobile Communication Architecture for Next-Generation Heterogeneous Wireless Systems” IEEE Radio Communications • June 2005

[4] S.Jamin, et al.,"A Measurement-Based Admission Control Algorithm for Integrated Service Packet Networks,” IEEE/ACM Transactions on Networking,vol.5,no.1,pp.56-70, Feb.1997. http://dx.doi.org/10.1109/90.554722

[5] Mark E.Crovella,and Azer Bestavros,"Self-Similarity in World Wide Web Traffic:Evidence and Possible Causes”IEEE/ACM Transaction on Networking,Vol.5, No.6,December 1997 http://dx.doi.org/10.1109/90.650143

[6] N. G. Duffield and N. O'Connell, "Large deviations and overflow probabilities for the general single server queue, with applications," in Mathematical Proceedings of the Cambridge Philosophical Society, pp. 363-374, 1995.

http://dx.doi.org/10.1017/s0305004100073709

[7] Paxon, V., Floyd, S.: Wide-area traffic: The failure of Poisson modeling. ACM/IEEE transactions on Networking 3(3) (1995) 226-244 http://dx.doi.org/10.1109/90.392383

[8] M. W. Garrett and W. Willinger, "Analysis, modeling and generation of self-similar VBR video traffic”, Proc. SIGCOMM 94, pp. 269-280, 1994. http://dx.doi.org/10.1145/190314.190339

[9] Basu, S., A. Mukherjee and S. RI. Klivansky, "Time- series models of Internet traffic," College of Computing Tech Report GIT-CC-95-27, July 1995.

[10] W.E. Leland, M.S. Taqqu, W. Willinger and D.V. Wilson (1993). Statistical analysis of high time-resolution Ethernet LAN traffic measurements. Proceedings of INTERFACE, 1993 